\title{
Effects of Health Education Intervention Using Health Belief Model on Haemoglobin Level Among Anaemic Pregnant Women in rural Malaysia: a quasi-experimental study
}

Hasneezah Hassan

Universiti Putra Malaysia

Rosliza Abdul Manaf ( $\square$ rosliza_abmanaf@upm.edu.my )

https://orcid.org/0000-0003-1488-1235

Salmiah Md Said

Universiti Putra Malaysia

Geeta Appannah

Universiti Putra Malaysia

Rozita Rosli

Universiti Putra Malaysia

Research article

Keywords: Anaemia, Pregnancy, Health Belief Model, Health education intervention, Compliance

Posted Date: July 19th, 2019

DOI: https://doi.org/10.21203/rs.2.11598/v1

License: (c) (i) This work is licensed under a Creative Commons Attribution 4.0 International License.

Read Full License 


\section{Abstract}

Background Anaemia in pregnancy is a problem threatening public health throughout the world especially in the developing countries. Current anaemia prevention programme in Malaysia includes oral iron supplementation and health education. However, the effect of the existing intervention in ensuring compliance to the subscribed regimen and the impact of nutrition education in enhancing dietary modification during pregnancy were minimal. This study aims to develop, implement and evaluate the effects of a theory-based health educational intervention using Health Belief Model (HBM) on haemoglobin level among anaemic pregnant women. Methods This is a quasi-experimental research with pre-post test design with control group involving 81 participants per group from two health clinics in Sepang, Malaysia. The primary outcome was haemoglobin level. Secondary outcome include knowledge on anaemia, HBM constructs, dietary iron intake and compliance towards iron supplementation. Both groups received routine antenatal care. Additionally, the intervention group received a theory-based health education intervention programme. Results The response rate in the intervention and control group were $83.9 \%$ and $82.7 \%$ respectively. Generalized estimating equations analysis showed that the intervention was effective in improving the mean haemoglobin level $(\beta=0.75,95 \% \mathrm{Cl}=0.52,0.99, P<0.001)$, the knowledge score $(\beta=1.42,95 \% \mathrm{Cl}=0.36,2.49, P=0.009)$, perceived severity score $(\beta=2.2,95 \% \mathrm{Cl}=1.02,3.39$, $\mathrm{P}<0.001)$ and increased proportion of high compliance level $(A O R=4.59,95 \% \mathrm{Cl}=1.58,13.35, \mathrm{P}=0.005)$. Conclusion Health education intervention based on the HBM is proven to be effective in improving the mean haemoglobin level, knowledge score, perceived severity score and compliance level of participants. The study results emphasized on the effectiveness of such an approach, therefore it is recommended that future educational interventions which aim at increasing preventive healthy behaviours in pregnant women could benefit from applying this model in primary health care settings. Trial registry Australian New Zealand Clinical Trials Registry with registration number of ACTRN12618001824257 (retrospectively registered)

\section{Background}

Anaemia in pregnancy is a serious global public health issue and poses a significant health problem among pregnant women [1]. The prevalence of anaemia in pregnant women was noted to be $38.2 \%$ across the world and $48.7 \%$ within countries located in the South East Asian region [2-3]. The prevalence of anaemia in pregnancy varies considerably because of the differences in socioeconomic conditions, lifestyles and health-seeking behaviours, across different cultures. Anaemia is emerging rapidly in developing countries. According to the WHO report of 2011, about $27 \%$ of the pregnant women in Malaysia have anaemia. The prevalence of anaemia in pregnancy has slightly increased to $29.3 \%$ in 2016 [4]. Therefore, it still constitutes a significantly moderate public health problem. Anaemia is defined as haemoglobin levels of less than $11 \mathrm{~g} / \mathrm{dl}$ for all trimesters [5]. Anaemia results from a number of causes, with iron deficiency being the primary contributor to anaemia and most widespread nutritional disorder [2]. The basic approaches use to prevent and control anaemia are to supplement with iron tablets and provide health education to the mothers [1]. 
In the context of Malaysia, the prevention of anaemia and the respective control strategies have been properly integrated into the maternal and child healthcare programmes initiated by the government, where iron supplementation is distributed for free to all pregnant women throughout all health clinics. The management of anaemia in pregnancy including a certain dosage of the iron supplements, with followups and referrals is outlined in the Malaysian Perinatal Care Manual [6]. However, the effectiveness of such healthcare education and motivation programmes given to pregnant women to ensure compliance with the treatment regiment, still confound the issue. Evidence provided by Thirukkanesh and Zahara [7] showed that, the compliance of the daily intake of vitamins and/or mineral supplements among pregnant women in Malaysia is only $49 \%$. Compliance was noted to be $47 \%$ among women in urban Selangor and $52 \%$ among women in rural Johor. These figures are considered to be very low when compared to the compliance of mothers in other developed countries such as Denmark which was about $80 \%$. The high figure has been attributed to the extended education programme of personal health promoted by the Danish National Board of Health [1]. Among the barriers causing women not to take these supplements include: fear of side effects and inadequate counselling about the benefits of the iron supplementations. These perceived barriers and benefits of compliance to the iron supplementation regime have the potential to improve through proper use of theories and models in the health education programme, health information and counselling provided [8].

Iron supplementation alone is not enough since the needs for calories and micronutrient such as iron increases during pregnancy. In Malaysia, all pregnant women also have given health education which aimed at empowering women with the importance of iron supplementations and increasing food intake rich in iron. Such kinds of health education are usually provided by nutritionists, nurses or doctors, during antenatal follow ups [6]. To date, there are no standard guidelines for a health education programme to follow; hence there are many variations of health education methods and materials being used in Malaysia [7]. Nonetheless, the effect of current health education to increase and modify to iron rich diet remain as an issue. In general, the diet of most South East Asians is mostly based on staples with little intake of animal-based foods, have low iron bio-availability and contain inhibitors of iron absorption [3]. In relation to this, Malaysians also have a low mean dietary iron intake [10]. Previous studies also reported that the intake of iron was inadequate and below the recommended nutrient intake of iron for their study population [11-12]. In Japan, a study revealed that $52 \%$ of the Japanese people had experienced anaemia in pregnancies. The cause is not only due to the mothers' physiological changes, but also their lifestyles [13]. This would imply drastic changes in the daily dietary iron intake among pregnant women during short period of time [1].

Apart from that, people have certain beliefs and perceptions and these can affect the pregnant mothers' behaviours towards the anaemia-preventive programmes introduced. The results gathered by Galloway et al. [14] of eight developing countries also showed that the women's perception of iron deficiency anaemia influenced its prevention and control. Their study detected that half of the women did not consider suffering from anaemia as it was not their priority concern. In addition to maternal perception, maternal knowledge of anaemia can be improved and corrected by giving regular health education during pregnancy [15-19]. 
In order to improve the status of anaemia among pregnant women, major changes in behaviour are needed, which centred on compliance with supplementation regimes and on dietary modification. It is important to also take note that each of these behavioural changes incur time, money and effort. Among the many health education programmes, it was noted that a theory-guided health education programme has a higher potential in facilitating voluntary actions that are conducive to health, thereby, increasing the higher adoption of health behaviour changes among individuals and communities alike. Various reviews have supported the beneficial effects of applying the Health Belief Model (HBM) in different health educational programmes. The HBM has served as a valuable tool in the field of educational evaluation to explain preventive health behaviour and has been applied as a guide for predicting health behaviour [2023]. The HBM framework will be able to emphasise on the relationship that exists between belief and health since the framework is based on the hypothesis that preventive health behaviours consist of personal beliefs [18]. The HBM framework includes several constructs like perceived susceptibility, perceived severity, perceived benefits, perceived barriers, cues to action and self-efficacy. There are several intervention studies that have been conducted to improve haemoglobin level, but only a small proportion has used established theories or model to guide the anaemia intervention. Those studies have proven that theory-based interventions were able to improve the haemoglobin level among anaemic pregnant women $[18,24-26]$.

Results from this study will add to the existing body of knowledge for relevant authorities. Furthermore, the literature review showed many gaps in terms of the effectiveness of anaemia treatment, whereby despite of iron supplementation, anaemia among pregnant women still persists. Literature reviewed also showed that there is no theory-based health education intervention programme which has been conducted in Malaysia which can be used to prevent anaemia in pregnancy. Not much has been explored on the perception of pregnant women regarding anaemia in pregnancy to be a problem. There is a need to further explore all these areas and strengthen the health education intervention to change the behaviours and ultimately improve the haemoglobin level during pregnancy. Since anaemia constitutes a significant public health problem in Malaysia and there is a lack of model-based studies in the field, the present study intends to develop, implement and evaluate the effects of a theory-based health educational intervention on the haemoglobin level among anaemic pregnant women. In particular, the pregnant women were those living in Sepang. If this intervention is found to be effective, it will help to reduce the prevalence and the common causes of anaemia and will improve the quality of life of mothers and their children.

\section{Methods}

\section{Study setting}

The study was carried out in two health clinics in Sepang District, which is located in rural area in Malaysia. Two health clinics in the district, namely, Salak and Dengkil Health Clinics were selected as the 
study location as they represented close similarities in terms of health system management including the type of health facilities that provide treatment to the respondents.

\section{Study design}

This study used a quasi-experimental study design with pre and post-test with control group involving two health clinics. Pregnant women in Salak Health Clinic were recruited as the control group and those at Dengkil Health Clinic were recruited as the intervention group. Both groups received routine antenatal care and practice which included iron supplementation from their respective clinics. Additionally, the intervention group was given theory-based health education intervention on anaemia in pregnancy. The study is conceptualized based on the Health Belief Model (HBM).

\section{Study population}

The sampling population is pregnant women in Salak and Dengkil Health Clinics diagnosed as having anaemia in pregnancy with haemoglobin $(\mathrm{Hb})$ level of less than $11.0 \mathrm{~g} / \mathrm{dl}$ and meet all the inclusion criteria. Subjects who met the following inclusion criteria were selected: Malaysian, pregnant woman with a $\mathrm{Hb}$ of less than $11 \mathrm{~g} / \mathrm{dl}$ until $7.0 \mathrm{~g} / \mathrm{dl}$ that was booked before 24 weeks of pregnancy and want to continue her antenatal check-up at these clinics. Known case of anaemia secondary to haematological disorders, severe anaemia $(\mathrm{Hb}<7 \mathrm{~g} / \mathrm{dl})$ and women with multiple pregnancies were excluded from the study. The sampling method was all eligible women who attended the antenatal clinics during the recruitment process were invited to participate in the study.

\section{Sample size estimation}

The sample size was calculated using the formula for two population mean formula for hypothesis testing [27]. $N=2 S 2(z(1-\alpha / 2)+z(1-\beta)) 2 /(\mu 1-\mu 2) 2$ with $S=$ pooled standard deviation, $Z 1-\alpha / 2=1.96$ (confidence level of $95 \%$, $\alpha$ is 0.05 ), $Z 1-\beta=0.84$ (power of $80 \%, \beta$ is 0.2 ), $\mu 1-\mu 2=$ estimated mean difference of two groups which $\mu 1$ and $\mu 2$ was selected from the study by Sennayake et al. [28]. Based on the above calculation, the minimum sample size needed consists of 81 pregnant women per group; after adjustment made for $20 \%$ attrition, eligibility of respondents and considering the design effect.

\section{Outcome measures}

The primary outcome of the study was haemoglobin level. The secondary outcome of the study was compliance level towards iron supplementation, dietary iron intake, knowledge on anaemia and HBM construct (perceived susceptibility, perceived severity, perceived benefit, and perceived barrier). The Hb level was measured before the 24 week of gestation (TO) and between week 35 and 37 of gestation in the 
third trimester ( $\mathrm{T} 1$ ). The difference between $\mathrm{T} 0$ and $\mathrm{T} 1 \mathrm{in}$ the intervention group was compared with the difference in the control group.

\section{Study questionnaire}

The questionnaires consist of the following five sections. Section one was socio-demographic factors and maternal obstetrics information. This section extracts the socio-demographic characteristics of age, ethnicity, education level, employment status and socio-economic status. Maternal obstetrics history includes gravida, parity, period of gestation, and spacing between children

Section two looks at participants' knowledge related to anaemia in pregnancy. The knowledge and perception questionnaire for this study was developed through adoption of structured questionnaire [2930]. A total of 20 items were applied to assess the structured knowledge questionnaire. Each question has three options, "Yes", "No", and "I don't know". Correct answers achieve one (1) mark and incorrect answers or "I don't know" achieve zero (0) marks. The total score for this section is 0 to 20 , with higher values indicating better knowledge towards anaemia in pregnancy. The content validity as assessed by content validity index, result was 0.70 . For reliability of knowledge section, Kuder-Richardson 20 was used, result was 0.81 .

Section three tests the participants' perceptions. The questionnaire was based on Health Belief Model including perceived susceptibility, perceived severity, perceived benefit and perceived barrier. Perceived susceptibility was assessed with 5 items, each rated on a 5-point Likert scale ranging from 'strongly agree' (5) to 'strongly disagree' (1). The total score for this section was 5-25 with higher values indicating better perceived susceptibility. Perceived severity consisted of 7 items, each rated on a 5-point Likert scale ranging from 'strongly agree' (5) to 'strongly disagree' (1). The total score for this section was 7-35 with higher values indicating better perceived severity. Perceived benefits were assessed with 5 items, each rated on a 5-point Likert scale ranging from 'strongly agree' (5) to 'strongly disagree' (1). The total score for this section was 5-25 with higher values indicating better perceived susceptibility. Perceived barrier consisted of 6 items, each rated on a 5-point Likert scale ranging from 'strongly agree' (5) to 'strongly disagree' (1). The total score for this section was 6-30 with higher values indicating poor perceived barrier. The content validity as assessed by content validity index, result was 0.75 . For reliability of perception section, Cronbach alpha was 0.77 .

Section four focussed on the level of compliance towards iron supplementation questionnaire. It consists of 4 questions that can measure participant level of compliance for iron supplementation [31]. Total score for this section was $0-4$ with score of 0 indicating high compliance level, score of 1-2 indicating medium compliance level and score of 3-4 indicating low compliance level towards iron supplementation.

Section five looks at participants' dietary iron intake. Three-day food record ( 2 weekdays and 1 weekend) was used to measure dietary intake [32]. Instructions were stated on the food record on how to measure 
servings without any leftovers of food. Pictures of household measurements such as spoon and cups were attached to the food records to facilitate serving size estimation. Also, other details such as method of cooking, ingredients used e.g. condiments and fat on meat were requested. Amount of food consumption including dietary iron by individuals were converted to milligrams by using dietary analysis software, Nutritionist Pro or Malaysian Food Composition Table. Data on dietary iron intakes were transferred from the Nutritionist Pro to the SPSS for statistical analysis. Dietary iron intake adequacy was assessed by comparison of energy and iron intake with the recommended nutrient intakes (RNI) for Malaysia pregnant women [10]. Dietary adequacy was considered achieved if the individual's mean intake met or exceeded $100 \%$ of the RNI.

\section{Intervention module}

The intervention module was developed through the process of consultations with a group of experts, studying relevant literature and received opinions from the community being served. The goal of this health educational intervention is for behavioural change. The module development was guided by the Health Belief Model (HBM). The HBM addresses four major components for compliance with recommended health action: perceived barrier of recommended health action, perceived benefits of recommended health action, perceived susceptibility of the disease and perceived severity of the disease.

\section{Intervention strategies}

This program was implemented using three strategies: Pre and post intervention assessment and health education intervention. The pre-test assessment was measured through study questionnaire given to the participating women before 24 weeks of gestation and followed by the health education intervention was held within 12 weeks (3 month). There is a variety of learning activities during that period that include health talks, small group discussions, poster presentation and pamphlets. Post intervention evaluation was measured between the $35^{\text {th }}$ to $37^{\text {th }}$ weeks of gestation in the third trimester.

\section{Baseline assessment}

Prior to starting the intervention programme, baseline or pre-test assessment need to be performed among the anaemic pregnant women at the selected health clinics prior to health education program to identify their baseline haemoglobin level, socio-demographic status, baseline status of their knowledge, perception towards anaemia in pregnancy, dietary iron status and compliance level. A set of selfadministrated study questionnaire was used to capture the baseline data. All participants must give their consent prior to answering the questionnaires and their answers are made confidential.

\section{Post intervention assessment}


After the completion of the twelve-week intervention period, the impact and outcome evaluation of the intervention programme was carried out using a set of questionnaires. The same set of questionnaires that was used in the pre-intervention assessment, except their socio-demographic status, was distributed to the participants. The post intervention haemoglobin level was taken from the participants' antenatal book. The outcome of the activity was to determine a post-intervention level of knowledge, perception and anaemia preventive behaviour after health education program.

\section{Health education intervention}

Health education interventions were implemented after a pre-intervention assessment has been conducted. The health education intervention periods was held within 12 weeks. There is a variety of learning activities during that period. The methods and material of the theory-based health intervention programme included health talk, small group discussion, poster presentation and pamphlets.

The health talk was given by the researcher on the topics such as introduction to anaemia in pregnancy, predisposing factor, sign and symptoms, complication, prevention of anaemia in pregnancy, knowledge on wrong perception of anaemia and iron supplementation and knowledge on the various sources of food that contain high iron. It is subsequently followed by a question and answer session or activity. In this activity, participants are able to ask anything regarding anaemia in pregnancy. The health talk was given once for each participant during the intervention period in form of power point presentation for about one hour. The outcome of the health talk would enable participants to gain knowledge on the facts of anaemia in pregnancy, complication of untreated anaemia and management of anaemia in pregnancy (iron supplementation and dietary iron intake) and its importance.

The second activity of the health education intervention programme was small group discussion. A session on small group discussion was conducted and facilitated by the researcher between two to four weeks after the health talk. The participants were divided into nine small groups with eight to ten participants in one group. Each group was encountered one session during the intervention period based on timing of the participants. This activity deals with the issues to enhance the compliance towards iron supplementation and to increase the dietary iron intake by identifying their barriers towards anaemia preventive behaviours. The outcome of the discussion would enable participants to understand the severity of anaemia in pregnancy, understand the susceptibility of anaemia in pregnancy, identify and overcome the barrier of not comply with iron supplement and dietary iron intake and identify the benefit of taking iron supplement and dietary iron intake. The participant also was provided with the checklist on compliance for iron supplementation. The time required to complete the group discussion session is within 60 minutes.

The materials used in the health education intervention programme are posters and pamphlets. The posters and pamphlets were designed to make teaching interesting and receptive. Two posters were used in this activity. A large poster (A1 size) was placed in the health clinic at all time during the intervention period. There was information regarding the complication of anaemia and importance of taking iron 
supplementation and consumption of iron rich food. In addition, the participants were given a small poster (A4 size) to bring home as their continuous learning material after the small group discussion session. The small poster was to provide guidelines of various iron rich food. A checklist on compliance towards iron supplementation was pasted in the maternal health record. Apart from that, a pamphlet also was distributed to the participants as a self-learning tool and continuous material that aimed at enriching their information about anaemia after the health talk or small group discussion. It also helped to deliver take home messages to the participants about anaemia in pregnancy. It was covered the information regarding the definition, causes, complications and prevention of anaemia in pregnancy and examples of food rich of iron. These materials were also aimed to maintain adherence towards anaemia preventive behaviour.

\section{Statistical analysis}

Data was analysed using IBM Statistical Package for Social Science (SPSS) version 25.0. Per protocol analysis was adopted as primary analysis in this study. Per protocol analysis were utilized for those completed all 2 data collection point and included in the final analysis.

Descriptive statistics using continuous data was analysed using means, standard deviation, median and inter-quartile range. While categorical data analysed using frequencies, percentage. Normality of the data was checked using histogram, Kolmogrov-Smirnov and Shapiro-Wilk normality test. An outcome variable was compared at the baseline and post-test. For within group difference, if data were assumed normal distributed data, parametric test such as Paired $t$ test was utilized. But if data were assumed non-normal distributed data, non-parametric tests such Wilcoxon Signed Ranks test was utilized. Apart from that, Mc Nemar Test was used to determine the association of two categorical related variables. For between groups difference, if data were assumed normal distributed data, parametric test such as Independent $\mathrm{t}$ test was utilized. But if data were assumed non-normal distributed data, non-parametric tests such MannWhitney $U$ test were utilized. Chi Square test and Fisher Exact were used to test the association between two categorical variables. Multivariable analysis was done using generalised estimating equations (GEE) to determine the effect of intervention on the primary and secondary outcome after adjusting for the covariates. The effect of intervention on changes in outcome measures was determined three months after the intervention period.

\section{Results}

A total of 171 participants were screened for eligibility. Out of these, 9 were excluded as they did not meet the inclusion criteria. A total of 162 anaemic pregnant women who met all the inclusion and exclusion criteria were invited to participate in this study. All 162 participants had completed their questionnaire at baseline giving a response rate of $100 \%$. However, only 135 participants completed all two time point data collection due to dropout and non-response. Hence, at three-month post intervention, $19 \%$ and $20 \%$ 
dropped out in the follow up at the intervention and control group respectively. The reasons for the study dropout are shown in Figure 1.

\section{[Insert Figure 1 here]}

The overall mean (SD) age of 162 respondents was 29years (4.95). The mean gestational week of participant in the intervention and control group was 21 week and 22 week. Majority of respondent in both groups booked their pregnancy during second trimester (96.9\%), had parity between 2 to 4 (60.5\%) and had gravida between 2 to 5 (68.5\%). There were no significant differences seen between the intervention and control groups in regards to their sociodemographic and maternal obstetric history as shown in Table 1.

\section{[Insert Table 1 here]}

At first model construct was compared between two groups at baseline. Result showed that baseline comparison on mean haemoglobin level, mean knowledge score, mean HBM construct score, high compliance level and mean dietary iron intake were found no significant different between two groups. For post intervention within group different, result showed that significant differences were seen in all model construct, except for the mean score of perceived benefit and mean dietary iron intake in the intervention group (Table 2).

\section{[Insert Table 2 here]}

Generalised estimating equation (GEE) was performed to determine the effect of the intervention on all model construct after adjusting the covariates. GEE was performed as GEE results are robust for correlated linear and binary outcome. Result showed that, health education intervention based on health belief model was effective in improving the mean haemoglobin level $(\beta=0.75,95 \% \mathrm{Cl}=0.52,0.99$, $P<0.001)$. For secondary outcome, health education intervention was effective in improving the mean knowledge score $(\beta=1.42,95 \% \mathrm{Cl}=0.36,2.49, \mathrm{P}=0.009)$, mean perceived severity score $(\beta=2.2,95 \% \mathrm{Cl}=$ $1.02,3.39, \mathrm{P}<0.001)$ and high compliance level $(\mathrm{AOR}=4.59,95 \% \mathrm{Cl}=1.58,13.35, \mathrm{P}=0.005)$ among study participant in the intervention group. GEE result tabulated in Table 3(a) to Table 3(d).

\section{[Insert Table 3(a) to 3(d) here]}

\section{Discussion}

This study implemented a theory-based health education intervention module, in which each component was discussed carefully. The Health Belief Model was chosen in this study as these models widely used in intervention programs aiming to changing behaviors towards preventive behaviour and most of the 
results had been proven successful. Intervention was guided by model which able to increase the knowledge, perception and lead to preventive behaviour of respondents and improve haemoglobin level.

The results show that theory-based health education intervention was effective on improving the mean haemoglobin level. The findings were similar with studies conducted in Indonesia, Saudi Arabia and India, where they reported the effectiveness of theory-based health education and found improvement in the mean haemoglobin level after their intervention [25,28,33-34]. At three months post-test, the difference in mean (SD) haemoglobin level between the intervention group and control group was $0.5 \mathrm{~g} / \mathrm{dl}$. Those differences in the haemoglobin of $\geq 0.5 \mathrm{~g} / \mathrm{dl}$ were also found in a study conducted by Widyawati et al. [33]. Those differences $(\mathrm{Hb} \geq 0.5 \mathrm{~g} / \mathrm{dl})$ were based on the women with higher supplementation who achieved an increase in the haemoglobin level around $0.5 \mathrm{~g} / \mathrm{dl}$ compared to those with lower supplementation [35]. This can be concurred by the participants in the intervention group who had falls in high score knowledge and HBM construct, and high compliance level towards iron supplementation as compared to control group.

For secondary outcomes, this study shows that the health education intervention was effective in improving the mean knowledge score, mean perceived severity score and high compliance level in the intervention group. For knowledge score, this study findings was similar to a studies done in Iran and Indonesia which reported that the health educational intervention were able to improve the knowledge level during the pregnancy after 3 month $[17,36]$. Mean perceived severity score was the highest mean score increased after intervention. Javaher et al. [37] also revealed that health education given makes them understand and belief of anaemia complication if they fail to comply with iron supplements and do not practice eating iron rich food. This finding is in agreement with another study done in Iran which reported that perceived severity score was significantly improved after implementation of health education $[18,26]$.

For high compliance level, this result is similar with a study conducted among anaemic pregnant women in India which reported that about $70.0 \%$ of pregnant women were compliant to iron supplementation after health education intervention was given and they became non-anaemic after 3 months [25]. Among the reasons of not complying with iron supplementation in this study was fear of side-effects and not interested to take the iron tablet, was reduced after the intervention. These results indicate that providing group consultations that focus on enhancing compliance towards iron supplementation is able to identify more barriers that can interfere with the practice and could propose strategies to increase compliance to pregnant women. This result reflects that the current study focused on imparting information and knowledge not only on anaemia but it also for subjects to assess their own perception, belief and behaviour according to the information given and make their decision on the changes they need to make. This could be helped by the participant in the intervention group had some level of education as compared to control group that make their receptive towards information better. The findings underscore the significance of health education intervention-based model. This result reflects that the improvement for mean haemoglobin level after intervention is most probably due to the high compliance level towards iron supplementation.

Page $11 / 26$ 
However, the health education intervention was not effective in improving the mean for perceived susceptibility, barrier, benefit and mean dietary iron intake score. These current results are not in line with the study conducted by Mariam et al. [18], Baharzadeh et al. [24] and Khorambadi et al. [26] which found that health education intervention given was effective in improving the mean perceived susceptibility score, barrier and benefit score. All of these studies used health education based on health Belief Model and similar teaching methods including group discussion, lecture and pamphlets. However, the time interval used to measure the effect of intervention was four months after intervention given; much longer as compared to the current study. This fact reflects that the current study time interval may be too short to detect the effect of intervention on the perceived susceptibility score. A three- month time interval was chosen due to time limitation, as post-test haemoglobin level was routinely done at respective health clinic as their requirement for management of anaemia in pregnancy at $35^{\text {th }}$ to $37^{\text {th }}$ gestational week.

For perceived benefit score, the current study was found to be not effective and this could be due to a 'ceiling' effect, as subjects in the intervention group had high mean perceived benefit score at baseline. The fact subjects had high scores for perceived benefit suggests that the mothers were knowledgeable regarding perceived benefit of taking iron supplementation and taking nutritious food over time, but still neglect to practice it. It would appear that if the pregnant women note that there are many types of iron rich food which they are able to choose from and are easy to prepare, these facts can be effective in improving the level of perceived benefits.

For perceived barrier score, this result was reflex as they had perceived barrier regarding types of iron rich food needed to be eaten and they still had poor diet rich iron at post-test. Another study done also reported a similar finding which found the unbalanced and poor diet was a part of perceived barrier [38]. Even though pregnancy is a good time for health education, but barrier to dietary change and selecting appropriate iron rich food take time to control. In addition, effective nutrition education would require several sessions and more focus on practical applications to achieve better results.

For mean dietary iron intake, the mean dietary iron intake of respondents at post-test was $24.8 \mathrm{mg}(9.3)$ and still below the Malaysian recommended iron intake (RNI) for pregnant women of $27 \mathrm{mg}$ per day. Findings are similar with a study by Mirnalini et al. [11] which reported that intake of iron were inadequate and below the RNI for pregnant women. Another study by Pick et al. [12] also reported that the mean daily intake of iron in pregnant women were below the recommended amount at $17 \mathrm{mg}$ per day. The current finding is contrary with the study conducted in Palestine and Iran that found effectiveness of their health education intervention whereby pregnant women had statistically significant improvement in dietary iron intake after intervention was given $[18,24,39]$. These findings suggest that dietary change and select appropriate iron rich food take time to occur. In addition, a major barrier for dietary behaviour found in this current study was the type of iron rich food needed to be eaten. It is suggested that effective nutrition education should require several sessions and concentration on the practical applications in order to achieve satisfactory results. 
A study by Jersey et al. [40] found that although knowledge played a key role for behaviour change, however, nutrition knowledge was inadequate and cannot lead to appropriate behaviour change. This fact reflects that although the knowledge was good, but motivation to make behaviour modification was not enough to take more dietary iron. Generally, Malaysians consume proportionately more carbohydrate but less fat and protein. This study also found similar findings whereby $54.4 \%$ of participants in the intervention group had consumed low protein and only $16.2 \%$ had enough fat consumption at posttest. Thus, the generally low intake of iron could be due to the lower protein intake as reported in this current study. With lower protein intake, the intake of micronutrients including iron was expected to decrease. Besides, most of the participants in the intervention group had low income status at less than RM3000 per month. This implies that the economic status of the pregnant mothers could have contributed to their diet because of their inability to purchase more protein-valuable products such as red meat which is high in iron, but which may be relatively expensive.

\section{Strength and limitation}

This study involved an extensive literature review on anaemia related issues, followed by a systematic review of eight research articles for the purpose of identifying the effective methods used for teaching as well as to determine the key components that were needed to make the intervention more effective. Apart from this, the current study also used a three-day food record as a measure to record the dietary iron intake of the pregnant women. The information gathered from this would have provided a better estimate of the participants' iron intake. The researcher also used generalised estimating equations in the multivariable analysis as it was more robust.

A quasi-experimental study design could be a limitation as it may not have a high impact as compared to a randomized control trial. Random assignment of groups cannot be performed because a large number of groups (clinics) are required to realize the full benefit of randomization [41]. Random allocation of the intervention was not possible because of practical and logistic constraints.

As we used a self-administered questionnaire to collect data, dishonesty of respondents can be an issue, but this was unavoidable due to limitation of human resource to gather data for this research. Time is a limiting factor in this study. as we used pregnant women as subjects and HBM-based education was only evaluated in a short period of time (within three months) after completion of intervention and maintenance of behaviour change which needed a longer follow-up. Self-selection bias may occur as no randomization was conducted in this study and may influence the evaluation outcomes. Apart from that, missing data due to protocol violations was among the barriers that occurred in this study.

\section{Conclusion}

Health education intervention based on the HBM is proven to be effective in improving the mean haemoglobin level, knowledge score, perceived severity score and compliance level of study participants. The study results emphasized the effectiveness of such an approach, therefore it is recommended that 
future educational intervention aimed at increasing preventive healthy behaviour in pregnant women could benefit from applying this model in primary health care settings.

\section{Abbreviations}

HBM: Health Belief Model; Hb: Haemoglobin level; WHO: World Health Organization; RNI: Recommended Nutrient Intake.

\section{Declarations}

\section{Ethics approval and consent to participate}

The Ethical approval was obtained from the National Medical Research Register (NMRR) -NMRR-17-17934162 on 29 March 2017, the Ethics Committee for Research Involving Human Subjects Universiti Putra Malaysia (UPM/TNCPI/RMC/1.4.18.2) on 18 April 2017 and the Selangor State Health Department (JNKS/KA/Q-712/04-01 JLd 5 (11) on 7 Jun 2017.

\section{Consent for publication}

Not applicable

\section{Availability of data and materials}

All data generated and analysed during this study is available with the corresponding authors and can be obtained upon request

\section{Competing interests}

The authors declare that they have no competing interests.

\section{Funding}

This research was funded by the Putra IPS Grant (GP- IPS/2017/9576900) from Universiti Putra Malaysia. 


\section{Authors' contributions}

$\mathrm{HH}$ and RAM conceptualized the study design. SMS provided expertise in the statistical analysis. GA advised on the nutritional assessment. $\mathrm{HH}$ conducted the intervention, collected and analyzed the data and wrote the manuscript. RAM, SMS, GA and RR reviewed the manuscript. All authors read and approved the final manuscript.

\section{Acknowledgements}

The authors also would like to thank the Department of Community Health Universiti Putra Malaysia for their institutional support throughout this study. This manuscript is part of the requirements for degree of Doctor of Public Health (DrPH).

\section{Authors' details}

${ }^{1}$ Doctor of Public Health Candidate, Department of Community Health, Faculty of Medicine and Health Sciences, Universiti Putra Malaysia, 43400 Serdang, Selangor

${ }^{2}$ Department of Community Health, Faculty of Medicine and Health Sciences, Universiti Putra Malaysia, 43400 Serdang, Selangor.

${ }^{3}$ Department of Nutrition and Dietetics, Faculty of Medicine and Health Sciences, Universiti Putra Malaysia, 43400 Serdang, Selangor.

${ }^{4}$ Department of Biomedical Sciences, Faculty of Medicine and Health Sciences, Universiti Putra Malaysia, 43400 Serdang, Selangor.

\section{References}

1. Millman N. Iron deficiency and anaemia in pregnant women in Malaysia-still a significant and challenging health problem. Journal of Pregnancy and Child Health. 2015; 2(3): 1-8.

2. World Health Organization. The Global Prevalence of Anaemia in 2011. WHO Report. 2011. Available from http://apps.who.int/iris/bitstream/10665/97892415 64960_eng.pdf. 
3. World Health Organization. Prevalence of anaemia among pregnant women. The World Bank data; 2016. Available from http://data.worldbank.org

4. National Health and Morbidity Survey Report, volume two; maternal and child health findings. Institute of public health, National Institute of Health; 2016.World Health Organization. Iron Deficiency Anemia: Assessment Prevention and Control: A Guide for Programme Mangers. Geneva. World Health Organization Publication. 2001. Available from http://www.who.int/nutrition/publications /micronutrie nts/anaemia_iron_deficiency/WHO _NHD_01.3/en/.

6. Ministry of Health Malaysia. Perinatal Care Manual 3rd Edition. Malaysia; 2013.

7. Thirukkanesh S, Zahara AM. Compliance to vitamin and mineral supplementation among pregnant women in urban and rural areas in Malaysia. Asia Pacific Journal of Nutrition. 2010; 9: 744-750.

8. Pallikadavath S, Foss M, Stones W. Antenatal care: provision and inequality in Rural North India. Social science and medicine. 2004; 59 (6): 1147-1158.

9. Noraini J, Jamaliah J, Norraliza MZ, Norizzati B, Roswati M, Hashim S. A study on the compliance of antenatal mothers to haematinics and outcome after structured interventions among antenatal mothers in the health clinics of two districts in Selangor, Unpublished study.

10. Ministry of Health Malaysia. Recommended nutrients intake for Malaysia, a report of the technical working group on nutritional guidelines. National Coordinating Committee on Food and Nutrition. Malaysia; 2005.

11. Mirnalini K, Zalilah MS, Yusof S, Tahir A, Siti HMD, Siti RD, Normah H. Energy and nutrient intakes: findings from the Malaysian adult nutrition survey (MANS). Malaysian Journal of Nutrition. 2008; 14(1): 1-24.

12. Pick ME, Edwards M, Moreau D, Ryan EA. Assessment of diet quality in pregnant women using the healthy eating Index. Journal of the American Dietetic Association. 2005; 105:240-6. 
13. Akase T, Hihara E, Uematsu K, Kodaka M, Tashiro S. Single center survey of the relationship between pregnancy anaemia and prepregnancy lifestyle. Yakugaku Zasshi. 2008; 1(28):1081-6.

14. Galloway R, Dusch E, Elder L, Achadi E, Grajeda R, Hurtado E, Favin M, Stephen C. et al. Women's perceptions of iron deficiency and anemia prevention and control in eight developing countries. Social Science and Medical Journal. 2002; 55(4):529-44.

15. Salama AM. Utilizing health belief model to enhance the preventive behaviour against iron deficiency anaemia among pregnant women. Journal of Nursing and Health Sciences. 2018; 7(4): 59-69.

16. Nimbalkar PB, Patel JN, Thakor N, Patni M. Impact of educational intervention regarding anaemia and its preventive measures among pregnant women: an interventional study. International Journal of reproduction, Contraception, Obstetrics and gynaecology. 2017; 6(12): 5317-5321.

17. Egryani NPR, Saktini F, Susilaningsih N, Puspitasari VD, Gumay AR. The effect of one to one counseling to pregnant women's knowledge about anemia in Semarang. Environmental Science Journal. 2016; 55:12-36.

18. Khoramabadi M, Dolatian M, Hajian S, Zamanian M, Taheripanah R, Sheikhan Z, Seyedi-Moghadam A. Effects of education based on health belief model on dietary behaviors of iranian pregnant women. Global Journal of Health Science. 2016; 8(2): 230-239.

19. Howiya S, Aziza IM, Lamiaa T. Effect of nutritional educational guidelines among pregnant women with iron deficiency anemia at rural areas in Kalyobia Governorate. Life Science Journal. 2012; 9(2): $1212-1217$.

20. A review of the use of the health belief model for weight management. Medsurg nursing. Academy of Medical-Surgical Nurses. 2007; 16(6): 363-6.

21. Chang C, Nayak S, Robert MS. Health beliefs about osteoporosis and osteoporosis screening in older women and men. Health education journal. 2007; 69(3):267-276. 
22. Sharifirad G, Tol A, Mohebi S. Effectiveness of nutrition education program based on health belief model compared with traditional training on the recommended weight gain in during pregnancy. Journal of Education and Health Promotion. 2013; 2: 15.

23. Glanz K, Rimer BK, \& Viswanath K. Health Behaviour and Health Education. Health Education. 2008 : (63). http://doi.org/10.1016/S0033-3506(49)81524-1.

24. Mariam A, El-guindi FK. Effect of nutritional interventions on anaemic pregnant women's health using health promotion model, The Medical Journal of Cairo University. 2010; 78(2): 109-118.

25. Noronha JA, Mphil N, Bhaduri A, Former ED, Bhat HV, Pro F. Interventional study to strengthen the health promoting behaviours of pregnant women to prevent anaemia in Southern India. Midwifery. 2013; 29(7): 35-41.

26. Baharzadeh K, Marashi T, Saki A, Javid AZ, Araban M. Using of health belief model to promote preventive behaviour againts iron deficiency anemia among pregnant women. Journal of Research \& Health. 2014; 7 (2): 754-762.

27. Lemeshow S, Hosmer DW, Klar J, Lwanga SK. Adequacy of sample size in health study. World Health Organization. John Wiley \& Sons. 1990

28. Sennayake HM, Premaratne SP, Palihawadana T, Wijeratne S. Simple educational intervention will Improve the efficacy of routine antenatal iron supplementation. The Journal of Obstetrics and Gynaecology Research. 2010; 36(3): 646-650.

29. Food and Agriculture Organization of United Nations. Guidelines for assessing nutrition related knowledge, attitude and practice. United Nations. 2014

30. Conner $\mathrm{M}, \&$ Norman P. Predicting health behaviour: research and practice with social cognition models. Second Edition. Open University Press; 2007. 
31. Morisky DE, Green LW, Levine DM. Concurrent and predictive validity of a self-reported measure of medication adherence. Medical Care Journal. 1986; 24:67-74.

32. Shahar S, Safii NS, Abdul Manaf Z, Haron H. Atlas of food exchanges \& portion sizes. Third Edition. MDC Publisher, Kuala Lumpur; 2015.

33. Widyawati W, Jans S, Bor HHJ, Dillen JV, Lagro-janssen ALM. The effectiveness of a new model in managing pregnant women with iron deficiency anaemia in Indonesia: a nonrandomized controlled intervention study, BIRTH: Issues in Perinatal Care Journal. 2015; 42(4): 337-345.

34. Badawy AS, Yakout SM, Taha N, Al-Salooly HA. Effect of iron supplementation and nutritional education among a group of anemic pregnant women on their perinatal outcome in riyadh. Journal of Current Research in Science. 2014:2(1):2322-500941.

35. Sloan NL, Jordan E, Winikoff B. Effect of iron supplementation on maternal hematologic status in pregnancy. The American Journal of Public Health. 2002;92(2): 288-293.

36. Kamalifard M, Mohammad Alizade Charandabi S, Ebrahimi Mamegani M, Asghari Jafabadi M. The knowledge, attitude and behaviour of pregnant women. Iranian Journal of Medical Education. 2012; 12(9): 686-97.

37. Javaheri TF, Nikpour S, Kazemi EA, Sanaie N, Shariat PA. The effect of education based on health belief model of women with urinary tract infection. International Journal of Community Based Nutrition and Midwifery. 2014; 2(1): 2-11.

38. Rothman RL, Mulvaney S, Elasy TA. Self-management behaviour, racial disparities and glycemic control among adolescent with type 2 diabetes. Journal of Paediatrics. 2008; 121(4): 912-9.

39. Araban M, Baharzadeh K, Karimy M. Nutrition modification aimed at enhancing dietary iron \& folic acid intake: an application of health belief model in practice. European Journal of Public Health. 2017; 1-6. 
40. Jersey S, Nicholson J, Callaway L, Daniel L. An observational study of nutrition and physical activity behaviours, knowledge and advice in pregnancy. BMC Pregnancy and childbirth. 2013; 13: 115. Doi: 1186/1471-2393-13-115.

41. Margaret AH, Courtney RL, Charles M, Adithya C. Selecting and improving quasi-experimental designs in effectiveness and implementation research. Annual review of Public health. 2018; 39(1): 5-25.Doi:.org/10.1146/annurev-publhealth040617-014128.

\section{Tables}

Table 1. Baseline comparison on socio-demographic characteristics and maternal obstetric history 


\begin{tabular}{|c|c|c|c|c|c|}
\hline \multirow[t]{2}{*}{ Variables } & \multicolumn{2}{|c|}{ Frequency, n (\%) } & \multirow[t]{2}{*}{$\mathrm{t}$} & \multirow[t]{2}{*}{$\chi^{2}(d f)$} & \multirow{2}{*}{$\begin{array}{c}\mathrm{P}- \\
\text { value }\end{array}$} \\
\hline & Intervention & Control & & & \\
\hline Age (mean) & $30.1(4.84)^{C}$ & $29.23(5.05)^{\mathrm{C}}$ & 1.11 & & $0.26^{a}$ \\
\hline \multicolumn{6}{|l|}{ Ethnic } \\
\hline Malay & $57(70.3)$ & $62(76.5)$ & & & \multirow{4}{*}{$0.11^{b}$} \\
\hline Chinese & $8(9.9)$ & $1(1.2)$ & & $5.80(3)$ & \\
\hline India & $12(14.8)$ & 14(17.3) & & & \\
\hline Others & $4(4.9)$ & $4(4.9)$ & & & \\
\hline \multicolumn{6}{|c|}{$\begin{array}{l}\text { Education level (Finished sec } \\
\text { school) }\end{array}$} \\
\hline Yes & $50(56.2)$ & $39(43.8)$ & & $3.01(1)$ & \multirow[t]{2}{*}{$0.08^{b}$} \\
\hline No & $31(42.5)$ & $42(57.5)$ & & & \\
\hline \multicolumn{6}{|l|}{ Employment status } \\
\hline Working & $58(54.7)$ & $48(45.3)$ & & $2.72(1)$ & \multirow[t]{2}{*}{$0.09^{b}$} \\
\hline Not working & $23(41.1)$ & $33(58.9)$ & & & \\
\hline \multicolumn{6}{|l|}{ Income } \\
\hline 0-2999 & $49(51.6)$ & $46(48.4)$ & & $0.22(1)$ & \multirow[t]{2}{*}{$0.63^{b}$} \\
\hline 3000 and above & $32(47.8)$ & $35(52.2)$ & & & \\
\hline \multicolumn{6}{|l|}{ Parity } \\
\hline Primipara & $34(42.0)$ & $27(33.3)$ & & $3.96(2)$ & \multirow[t]{2}{*}{$0.13^{b}$} \\
\hline $2-4$ & $47(58.0)$ & $51(63.0)$ & & & \\
\hline$\geq 4$ & $0(0)$ & $3(3.7)$ & & & \\
\hline \multicolumn{6}{|l|}{ Gravida } \\
\hline Primigravida & $26(32.1)$ & $20(24.7)$ & & $1.20(2)$ & $0.54^{\mathrm{b}}$ \\
\hline $2-5$ & $53(65.4)$ & $58(71.6)$ & & & \\
\hline 6 and above & $2(2.5)$ & $3(3.7)$ & & & \\
\hline \multicolumn{6}{|l|}{ Gestational week } \\
\hline $\begin{array}{l}1^{\text {st }} \text { trimester } \\
(<12 \text { week })\end{array}$ & $1(1.2)$ & $4(5.0)$ & & & $0.18^{\mathrm{d}}$ \\
\hline $\begin{array}{l}2^{\text {nd }} \text { trimester } \\
(12 \text { to }<28 \text { week })\end{array}$ & $80(98.8)$ & $76(95.5)$ & & & \\
\hline \multicolumn{6}{|l|}{ Birth spacing } \\
\hline Less than 24 month & $24(47.1)$ & $24(41.4)$ & & $0.35(1)$ & $0.55^{\mathrm{a}}$ \\
\hline 24 month and more & $27(52.9)$ & $34(58.6)$ & & & \\
\hline
\end{tabular}

$\mathrm{a}_{\text {Independent } \mathrm{t} \text { test, }} \mathrm{b}^{\mathrm{b}}$ Chi-square test, ${ }^{\mathrm{C}}$ Mean (SD), $\mathrm{d}_{\text {Fisher exact test. }}$

Table 2. Change in mean haemoglobin level, knowledge score, HBM construct score, dietary iron intake score and high compliance level in the both groups 


\begin{tabular}{|c|c|c|c|c|c|c|}
\hline Outcome & $\overline{\mathrm{Me}}$ & SD) & Mean & $95 \%$ CI & $t(d f)$ & P-Value \\
\hline & Baseline & Post test & & & & \\
\hline
\end{tabular}

\section{Haemoglobin}

level $^{\mathrm{a}}$

$\begin{array}{lcccccc}\text { Intervention } & 10.00 & 11.76 & 1.75 & 1.56,1.94 & 18.07 & <0.001^{*} \\ & (0.69) & (0.51) & & & (66) & \\ \text { Control } & 10.22 & 11.27 & 1.04 & 0.88,1.19 & 13.47 & <0.001^{*} \\ & (0.52) & (0.40) & & & (67) & \end{array}$

Knowledge score

$\begin{array}{lcccccc}\text { Intervention }^{\mathrm{b}} & 15.00 & 19.00 & -6.63^{\mathrm{d}} & & & <0.001^{*} \\ & (3.50)^{\mathrm{C}} & (1.00)^{\mathrm{c}} & & & & \\ \text { Control }^{\mathrm{a}} & 15.58 & 17.85 & 2.26 & 1.57,-2.95 & 6.57(67) & <0.001^{*} \\ & (3.45) & (1.84) & & & & \end{array}$

\section{Perceived}

susceptibility

score $^{\text {a }}$

$\begin{array}{lcccccc}\text { Intervention } & 17.89 & 19.33 & 1.43 & 0.65,2.22 & 3.66(66) & <0.001^{*} \\ & (2.52) & (1.72) & & & & \\ \text { Control } & 18.01 & 19.17( & 1.16 & 0.58,1.73 & 4.02(67) & <0.001^{*} \\ & (1.98) & 2.22) & & & & \end{array}$

\section{Perceived}

severity score ${ }^{a}$

$\begin{array}{lllllll}\text { Intervention } & 26.89(3.73) & 30.25(2.92) & 3.35 & 2.43,4.28 & 7.26(66) & <0.001^{*} \\ \text { Control } & 26.30(3.42) & 27.42(2.25) & 1.11 & 0.31,0.91 & 2.79(67) & 0.007^{*}\end{array}$

Perceived

benefit score ${ }^{a}$

\begin{tabular}{llllllc} 
Intervention & 22.35 & 23.05 & 0.70 & $-0.06,1.46$ & $1.83(66)$ & 0.07 \\
& $(2.56)$ & $(1.95)$ & & & & \\
Control & 21.51 & 22.67 & 1.16 & $0.54,1.77$ & $3.77(67)$ & $<0.001^{*}$ \\
& $(2.47)$ & $(2.24)$ & & & & \\
\hline
\end{tabular}

\section{Perceived}

barrier score ${ }^{a}$

$\begin{array}{lcccccc}\text { Intervention } & 24.50(4.04) & 23.52(4.42) & -0.98 & -1.94,-0.02 & -2.05(66) & 0.04^{*} \\ \text { Control } & 24.16(3.82) & 23.15(5.69) & -1.01 & -2.26,0.23 & -1.61 & 0.11\end{array}$

\section{Level of}

Compliance

Intervention $^{\mathrm{e}}$

Low

Medium

High

$\begin{array}{ll}15(22.7) & 7(10.6) \\ 41(62.1) & 30(45.5) \\ 10(15.2) & 29(43.9)\end{array}$

$0.002^{*}$ 
Control $^{\mathrm{e}}$

Low 15(22.1)

17(25.0)

0.64

medium

42(61.8)

40(58.8)

High

11(16.2)

11(16.2)

Dietary iron

intake score ${ }^{a}$

Intervention

22.96

(10.38)

24.81

(9.39)

Control

23.17

23.25

1.84

$-1.51,5.203 .66(66)$

0.27

(8.21)

(9.95)

${ }^{a}$ Paired t test, ${ }^{b}$ Wilcoxon signed rank test, ${ }^{\mathrm{C}}$ Median(IQR), ${ }^{\mathrm{d}} \mathrm{Z}$ statistic, ${ }^{\mathrm{e}}$ McNemar Test, *Significant at $\mathrm{P}<0.05$

Table 3(a): GEE analysis of mean haemoglobin level

\begin{tabular}{|c|c|c|c|c|c|}
\hline \multirow[t]{2}{*}{ Variable } & \multirow[t]{2}{*}{ B } & \multirow[t]{2}{*}{ SE } & \multicolumn{2}{|c|}{$95 \% \mathrm{CI}$} & \multirow[t]{2}{*}{ P-Value } \\
\hline & & & Lower & Upper & \\
\hline Trial group & & & & & \\
\hline Intervention & -0.232 & 0.104 & -0.436 & -0.028 & $0.026^{*}$ \\
\hline Control $^{\mathrm{a}}$ & & & & & \\
\hline Timepoint & & & & & \\
\hline 3 month & 1.004 & 0.073 & 0.860 & 1.149 & $<0.001^{*}$ \\
\hline Baseline $^{\mathrm{a}}$ & & & & & \\
\hline Trial group $\mathrm{x}$ timepoint & 0.758 & 0.121 & 0.521 & 0.995 & $<0.001^{*}$ \\
\hline
\end{tabular}

*Significant at $\mathrm{P}<0.05,{ }^{\mathrm{a}}$ Reference group, GEE adjusted for group, time, birth spacing, gestational week

Table 3(b): GEE analysis of knowledge score 


\begin{tabular}{llcl}
\hline Variable & B & SE $\frac{\text { 95\% CI P -Value }}{\text { LowerUpper }}$ \\
\hline
\end{tabular}

Trial group

Intervention $\quad-0.5760 .578-1.7090 .558 \quad 0.320$

Control $^{\mathrm{a}}$

Timepoint

3 month

$2.2650 .3421 .5942 .935<0.001^{*}$

Baseline $^{\mathrm{a}}$

Trial group x timepoint $1.4290 .5430 .3652 .494 \quad 0.009^{*}$

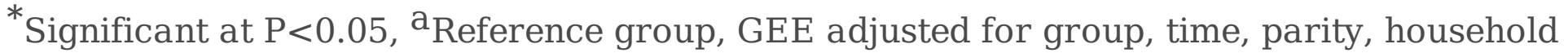
income, birth spacing.

Table 3(c): GEE analysis of mean perceived severity score

\begin{tabular}{|c|c|c|c|}
\hline Variable & SE & $\frac{95 \% \text { CI }}{\text { LowerUpper }}$ & P-Value \\
\hline Trial group & \multirow{3}{*}{\multicolumn{2}{|c|}{$\begin{array}{l}0.8590 .6042-0.3252 .043 \\
0^{a}\end{array}$}} & \\
\hline Intervention & & & 0.155 \\
\hline Control $^{\mathrm{a}}$ & & & \\
\hline Timepoint & & & \\
\hline 3 month & 1.1180 .397 & 0.3391 .896 & $0.005^{*}$ \\
\hline $\begin{array}{l}\text { Baseline }^{\mathrm{a}} \\
\text { Trial group x }\end{array}$ & $\begin{array}{l}0^{\mathrm{a}} \\
2.2060 .6\end{array}$ & 02 & \\
\hline
\end{tabular}

*Significant at $\mathrm{P}<0.05,{ }^{\mathrm{a}}$ Reference group, GEE adjusted for group, time, age, household income, status, parity.

Table 3(d): GEE analysis of high compliance level

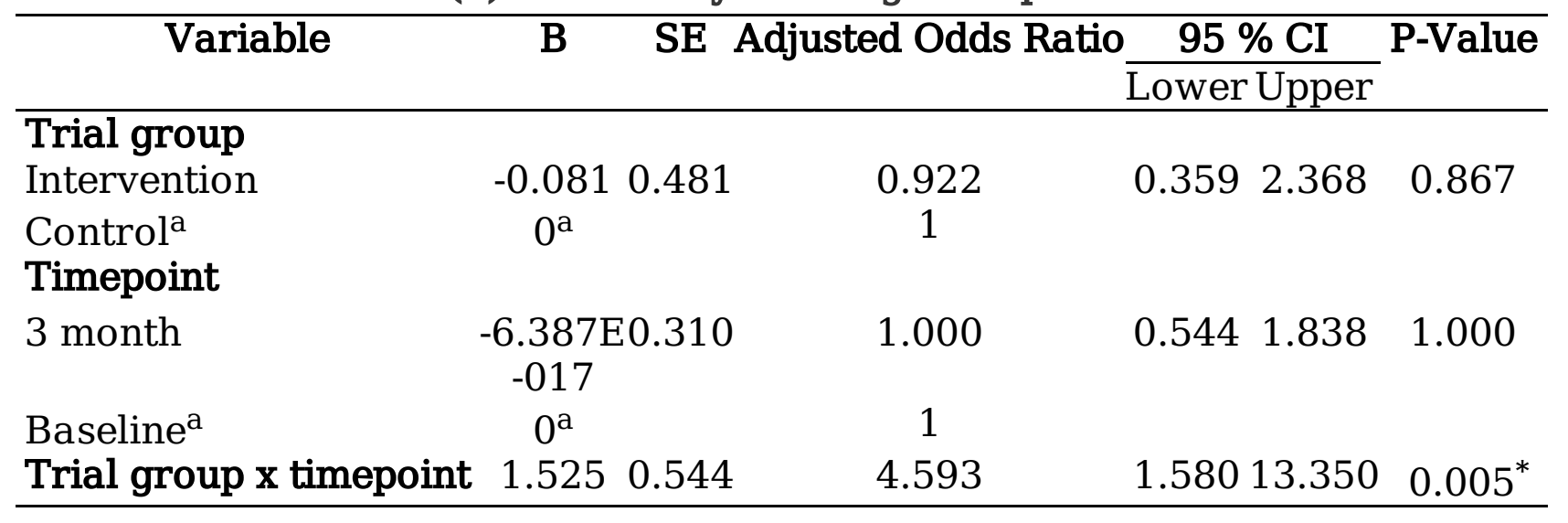

* Significant at $\mathrm{P}<0.05$, a Reference group, GEE adjusted for group, time, birth spacing. 


\section{Figures}

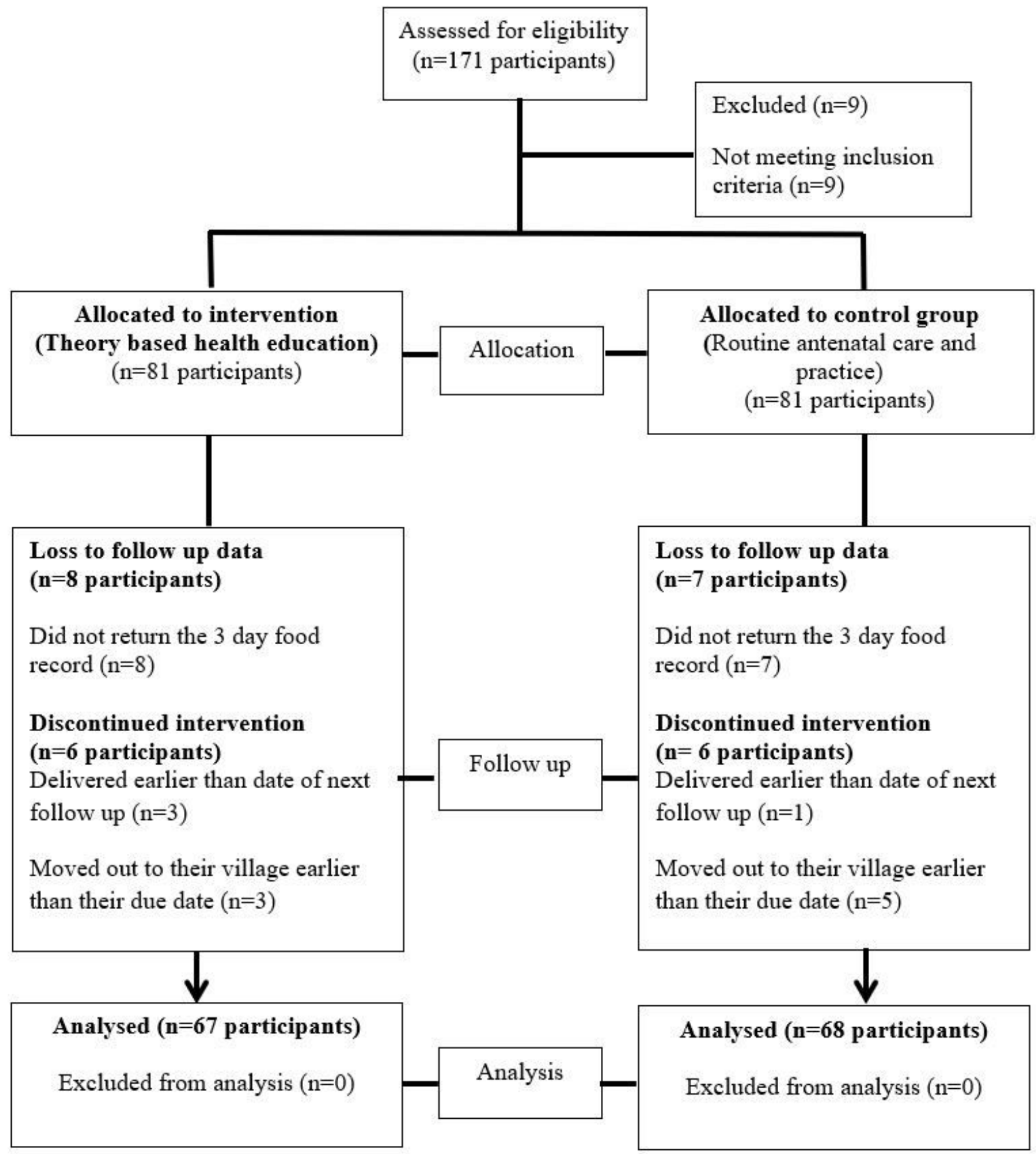


Figure 1

Flow Chart of Diagram of the Study.

\section{Supplementary Files}

This is a list of supplementary files associated with this preprint. Click to download.

- WRITTENINFORMEDCONSENT.doc

- StudyquestionnairesEnglish.doc 